\title{
Postpartum-Isolated Native Pulmonic Valve Methicillin-Resistant Staphylococcus aureus Infective Endocarditis Complicated by Pelvic Abscess, Clavicle Osteomyelitis, and Polyarticular Septic Arthritis
}

\author{
Daniela Navarrete $\mathbb{D}^{1},{ }^{1}$ David Hannibal, ${ }^{2}$ Sachin M. Patil, ${ }^{3}$ Tarang Pankaj Patel, \\ and William Roland ${ }^{3}$ \\ ${ }^{1}$ Department of Medicine and Pediatrics, PGY4 Resident, University of Missouri Hospital and Clinic, 1 Hospital Dr, Columbia, \\ MO 65212, USA \\ ${ }^{2}$ University of Missouri School of Medicine, MS 4, University of Missouri Hospital and Clinic, 1 Hospital Dr, Columbia, \\ MO 65212, USA \\ ${ }^{3}$ Department of Medicine, Division of Infectious Disease, University of Missouri Hospital and Clinic, 1 Hospital Dr, Columbia, \\ MO 65212, USA \\ ${ }^{4}$ Department of Medicine, Division of Pulmonary, Critical Care and Environmental Medicine, \\ University of Missouri Hospital and Clinic, 1 Hospital Dr, Columbia, MO 65212, USA
}

Correspondence should be addressed to Daniela Navarrete; dani20@umbc.edu

Received 11 August 2020; Revised 4 October 2020; Accepted 24 October 2020; Published 7 November 2020

Academic Editor: Antonella Marangoni

Copyright (c) 2020 Daniela Navarrete et al. This is an open access article distributed under the Creative Commons Attribution License, which permits unrestricted use, distribution, and reproduction in any medium, provided the original work is properly cited.

\begin{abstract}
Isolated native pulmonic valve infective endocarditis (IE) is a rare occurrence. The most commonly involved valves in injection drug users are the tricuspid valve followed by mitral and then aortic valves. Most reported cases of methicillin-resistant Staphylococcus aureus (MRSA) IE involve multiple valves. Isolated involvement of the pulmonic valve in IE is infrequent, especially in intravenous drug users or patients with indwelling catheters, prosthetic valves, or implantable cardiac devices. Here, we report a young postpartum female patient with isolated native pulmonic valve MRSA IE with MRSA bacteremia and history of active injection drug use. A PubMed literature review revealed a single described prior case report in a postpartum female. The patient's clinical course was complicated by a large native pulmonic valve vegetation, septic pulmonary emboli, pelvic abscess, polyarticular septic arthritis, and clavicular osteomyelitis. The patient underwent bioprosthetic pulmonic valve replacement and finished six weeks of intravenous vancomycin for complete recovery.
\end{abstract}

\section{Introduction}

The opioid crisis has become an increasingly prevalent public health and medical issue in the United States over the past 15 years [1]. In 2016, nearly 43,000 people died from opioid-related deaths, which is expected to rise to almost 100,000 per year [1]. Opioid addiction can be secondary to prescription opioids or illicit drugs. IE among injection drug users is approximately $2 \%$ to $5 \%$ per year and responsible for up to $20 \%$ of hospital admissions with a mortality of $10 \%$ [2].
The opioid crisis places an economic health burden, and therefore, addiction treatment and emphasis on addiction medicine are essential.

IE in injection drug use most commonly involves the tricuspid valve followed by the aortic and mitral valves. Pulmonic valve IE is an infrequent entity, and most of these cases do not have severe complications such as sepsis from septic emboli [3]. We present an injection drug user with methicillin-resistant Staphylococcus aureus (MRSA) bacteremia and pulmonic valve IE complicated by septic emboli 
to lungs, polyarticular septic arthritis. There is higher mortality in patients with both IE and septic arthritis [4], and injection drug users are at risk. Our case demonstrates that in injection drug users with bacteremia and IE, a thorough evaluation of different concurrent infections should be done. Musculoskeletal complaints should prompt further imaging.

\section{Case Presentation}

A 35-year-old white female presented to the emergency room for severe midsternal chest pain and left shoulder pain that started the previous day. She had a recent vaginal delivery at home approximately ten weeks before this presentation and had ongoing intravenous drug use. Since the birth at home, she had lower abdominal pain but failed to seek medical attention. She also reported two to four weeks of generalized malaise, fever, and chills. She was febrile at the emergency room, with tachycardia of 105 beats per minute, normal blood pressure, and oxygen saturation $>90 \%$ on room air. Based on her clinical history and physical examination findings such as tachycardia and severe lower abdominal tenderness, acute pulmonary embolism was a concern. She underwent a computed tomography (CT) scan of the chest, abdomen, and pelvis for further evaluation. Imaging revealed multiple small pulmonary emboli and findings concerning septic arthritis of the pubic symphysis and edema of the left clavicle. Based on her clinical presentation and risk factors, IE was suspected. Therefore, she was transferred to our hospital for further management.

On arrival at our facility, the patient had a fever of 38.8 ocelsius, tachycardia of 125 beats per minute, blood pressure of $129 / 79 \mathrm{mmHg}$, and oxygen saturation of $93 \%$ on room air. Labs revealed leukocytosis of $19,000 / \mathrm{mL}$ with neutrophil predominance and normal renal and hepatic functions. Labs were significant for hypoalbuminemia and elevated inflammatory markers, including ESR (Erythrocyte sedimentation rate) of $100 \mathrm{~mm} / \mathrm{Hr}$ and CRP (C-reactive protein) of $27 \mathrm{mg} / \mathrm{dL}$. Her urine drug screen was positive for amphetamines and opiates, and she admitted to the daily use of heroin and methamphetamine. On physical examination, she was alert and oriented but appeared to be in painful distress, expressing significant abdominal and left shoulder pain. Lungs were clear on auscultation, whereas on the cardiac exam, a diastolic murmur in the pulmonary area was heard. She had severe suprapubic area tenderness on palpation. The left shoulder limited range of motion with no spine tenderness was appreciated on musculoskeletal examination. Left antecubital and buttock areas showed the presence of multiple needle marks with no signs of infection. A portable chest X-ray disclosed diffuse lung opacities suspicious of septic emboli (Figure 1). Empirically intravenous (IV) vancomycin and ceftriaxone were initiated for suspected IE treatment. The transthoracic echocardiogram (TTE) demonstrated an extensive mobile $3.7 \times 0.5 \mathrm{~cm}$ vegetation on the pulmonary valve with moderate to severe pulmonic regurgitation (Figure 2). The patient refused a transesophageal echocardiogram (TEE) despite multiple requests. The cardiothoracic surgery team review of the TTE was negative for any left-sided IE stigmata.

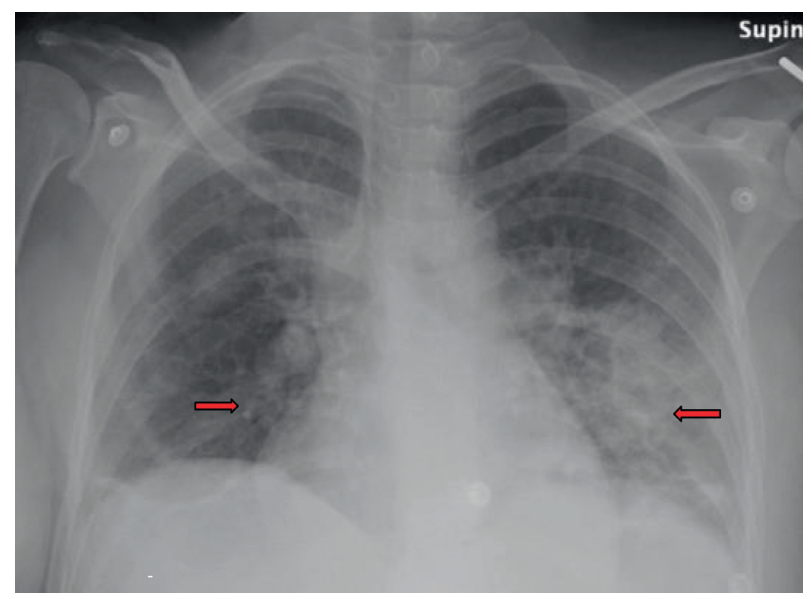

Figure 1: Chest X-ray revealed diffuse bilateral lower lobe predominant airspace opacities with nodularity.

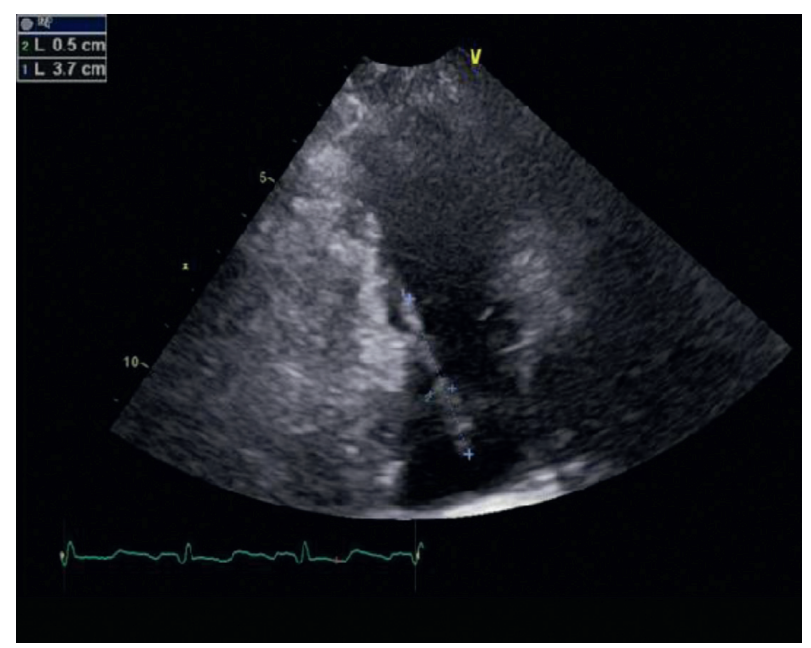

Figure 2: Transthoracic echocardiogram revealed extensive mobile $3.7 \times 0.5 \mathrm{~cm}$ vegetation in the pulmonary valve with moderate to severe pulmonic regurgitation mobile $3.7 \times 0.5 \mathrm{~cm}$ vegetation in the pulmonary valve with moderate.

Magnetic resonance imaging (MRI) of the left shoulder revealed acromioclavicular joint septic arthritis and abnormal marrow edema in the distal clavicle concerning for osteomyelitis (Figure 3). A subcentimeter abscess in the region of subacromial bursa was also seen (Figure 3). Pelvis MRI showed peripherally enhancing fluid collection from pubic symphysis compatible with septic arthritis and erosion in the left pubic body without confluent marrow replacement to indicate osteomyelitis and bilateral hip joint effusions (Figure 4). Blood cultures returned positive for MRSA within 12 hours, and they remained positive from the first day of hospitalization until day 7. The mean inhibitory concentration of vancomycin for MRSA was 1 . The positive blood cultures resulted in the de-escalation of antibiotics to vancomycin alone.

The patient underwent irrigation and debridement of the pelvic abscess and left shoulder joint. The cultures from the fluid aspiration of the left acromioclavicular joint and pubic 


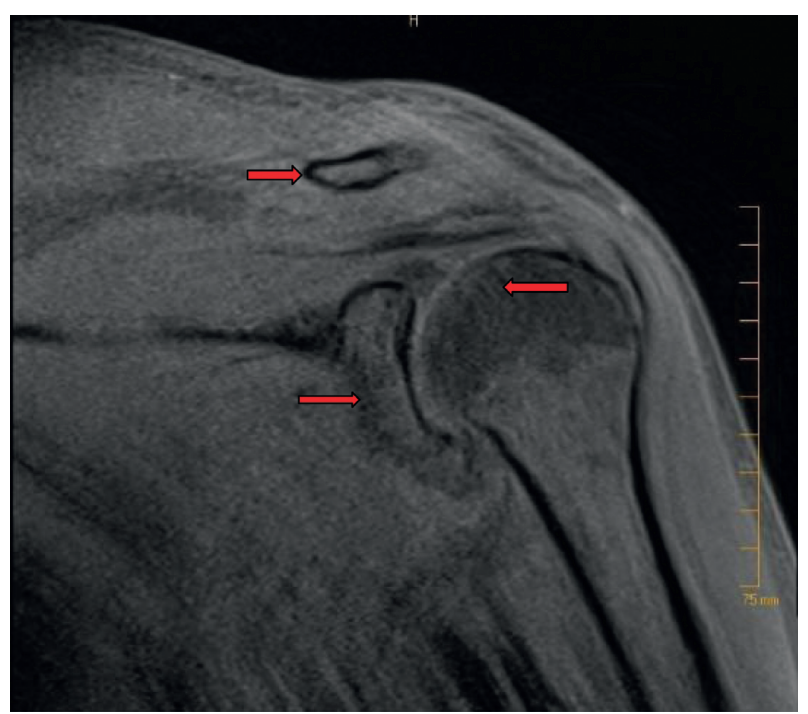

FIgURE 3: MRI left shoulder revealed acromioclavicular joint synovitis with periarticular soft tissue edema consistent with septic arthritis and distal clavicle marrow edema suggestive of osteomyelitis. A subcentimeter abscess in the subacromial bursa was also noted.

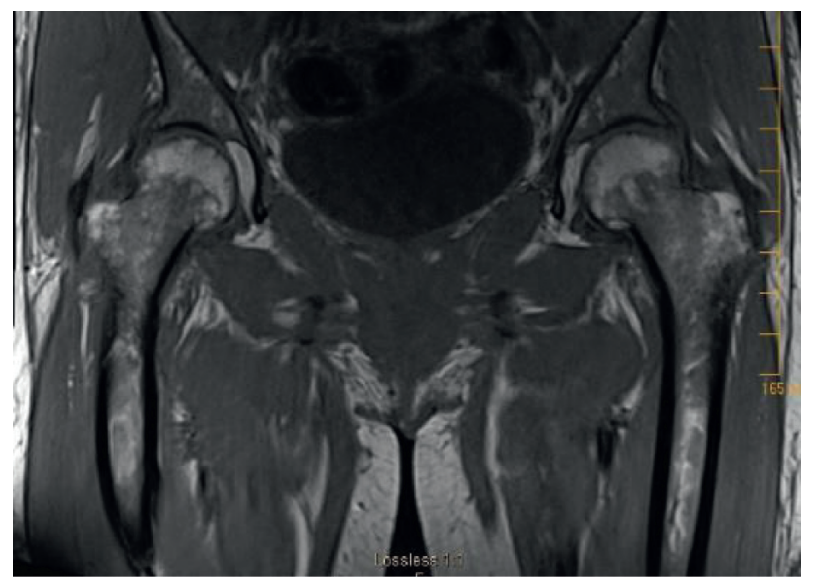

FIGURE 4: MRI pelvis revealed peripherally enhancing fluid collection extending into the adjacent soft tissues from the pubic symphysis consistent with septic arthritis. Left pubic body erosion without confluent marrow replacement indicates osteomyelitis. It also shows bilateral hip joint effusions with nonspecific left hip synovitis.

symphysis grew MRSA confirming septic arthritis. On the 13th day of hospitalization, she underwent bioprosthetic pulmonic valve replacement and pulmonary artery reconstruction with autologous pericardial patch placement. Intraoperative echocardiogram did not reveal any left-sided IE stigmata. The special stain for fungal organisms was negative. Cultures from the valve were not sent due to positive blood and tissue cultures from the joint aspirations. Histopathology of the pulmonic valve vegetation revealed a partially necrotic cardiac valve with septic vegetations, numerous Gram-positive cocci in clusters, and pairs. After one week following cardiac valve surgery, she had dental extraction of the remaining upper teeth to optimize dental hygiene. After valve replacement, she was started on warfarin with an international normalized ratio goal of 2-3.

Due to the history of active ongoing intravenous drug abuse, outpatient parenteral antibiotics were deferred. Instead, the patient received intravenous antibiotics, inpatient, until completion of her antibiotic course. She completed the IE treatment with six weeks of vancomycin. Inpatient pharmacy managed the vancomycin dosing based on the trough levels. Vancomycin dosing was three times a day, and the average dose ranged from $750 \mathrm{mg}$ to $1250 \mathrm{mg}$ over six weeks. Her mean vancomycin trough levels ranged from 15 $\mathrm{mcg} / \mathrm{mL}$ to $18 \mathrm{mcg} / \mathrm{mL}$. Upon completing antibiotic therapy, the ESR had decreased to $32 \mathrm{~mm} / \mathrm{Hr}$ and CRP decreased to $1.32 \mathrm{mg} / \mathrm{dL}$. Before being discharged home, she underwent an evaluation by psychiatry for suboxone treatment. She was discharged home in a stable condition. Unfortunately, she was lost to the outpatient clinic follow-up and repeat imaging to ensure that the infection resolution could not be obtained.

\section{Discussion}

IE is an infection of the endocardium or a prosthetic valve surface associated with a one-year mortality rate of up to $40 \%$ and an in-hospital mortality rate of about $20 \%$ [5]. These alarming statistics make this an important topic in healthcare. The outcomes have not improved substantially over the past 25 years, despite many advances in medical and surgical treatment [6]. Fortunately, IE is a rare disease with an incidence of 15 per 100,000 people in the United States in 2011 [7]. However, with the rising opioid crisis and injection drug use as a contributing fuel, IE cases will increase and place a burden on healthcare costs due to the need for hospitalization. An emphasis on the field of addiction medicine for management is one of the most vital aspects of overcoming the burden.

Our case involves the uncommon occurrence of pulmonic valve IE in a patient with intravenous drug use. In injection drug users, $60 \%$ of cases of IE occur on the tricuspid valve, and in about $40 \%$ of cases, the left heart valves alone are affected. The pulmonic valve is rarely involved, and this may be due to the low-pressure gradient and low wear and tear of this valve [8]. In all patient populations with infective endocarditis, pulmonary valve infections account for $\leq 2 \%$ of cases [9].

Most cases of pulmonary valve IE occur in children with congenital heart disease [3]. As a result of pulmonic valve IE being so rare, the diagnosis can be easily missed. A significant number of patients with pulmonic valve IE have pulmonary symptoms such as dyspnea, chest pain, and cough [10]. There is often a delay in diagnosis due to the lack of typical signs and symptoms seen with mitral or aortic endocarditis [3]. Modified Duke criteria facilitate pulmonic valve IE determination, and further evaluation includes a thorough physical exam, TEE, and blood cultures [11, 12].

In our patient, the pulmonary valve vegetation was visible on a TTE due to its large size. Although right-sided IE has a better prognosis than left-sided, it should not be neglected as the potential pulmonary emboli and IE 
complications seen in our patient can be fatal. Therefore, it is essential to pursue a TEE in patients with suspected infection and significant risk factors for IE, like injection drug use. IE and Staphylococcus aureus (S. aureus) bacteremia can become further complicated by infectious foci at any site. Our patient had a pelvic abscess, clavicular osteomyelitis, and polyarticular septic arthritis. It is rare for patients with IE to have septic arthritis with rates ranging from 5 to $31 \%$ [4]. S. aureus is one of the most commonly isolated organisms from synovial fluid in these patients, as was seen in our case, and intravenous drug use is a risk factor for both IE and septic arthritis [4].

Treatment of IE is $4-8$ weeks of intravenous antibiotics after the offending bacteria is identified [13]. The offending bacteria are often $S$. aureus, coagulase-negative staphylococci, or Group B streptococci [3]. Surgery is an option in patients with progressive heart failure, an infection resistant to antibiotics, complications such as abscess formation or recurrent pulmonary embolism, or vegetation greater than $2 \mathrm{~cm}[12,13]$. Our patient had pulmonary valve replacement due to the vegetation size and for source control.

Our case of pulmonic valve IE involved a patient during her postpartum period. IE is rare and life-threatening during pregnancy [14]. The calculated maternal mortality is $11.1 \%$, and the fetal mortality rate is $14.3 \%$ [14]. The mortality risk has improved most likely due to maternal-fetal monitoring, new surgical techniques, and nontoxic and effective antimicrobial agents [14]. Pregnant patients' risk factors, microbiology, and IE complications are similar to those of nonpregnant patients. During pregnancy, risk factors include injection drug use, congenital heart disease, and less commonly rheumatic heart disease [14]. Rheumatic heart disease has decreased, and the number of cases due to injection drug use has increased markedly. Streptococcal species are the most common pathogen in pregnant patients, followed by staphylococcal species [14]. However, rightsided infective endocarditis is more likely to be staphylococcal species [14].

On review of the PubMed literature, ours is the second case of isolated native pulmonic valve MRSA IE. Interestingly, the first reported case also was seen in a 19-year-old postpartum female [15]. Pulmonary, central nervous system, and systemic emboli are common complications [14]. The treatment for IE in pregnant women most commonly consists of a multidrug antibiotic regimen [14]. Selected patients may need surgical intervention and antibiotics. The surgical options include a valve replacement or valvuloplasty [14].

\section{Conclusion}

This case demonstrates IE's unique presentation due to isolated native pulmonic valve disease with concurrent septic arthritis and osteomyelitis during the postpartum period. Our patient presented with polyarticular septic arthritis and left clavicular osteomyelitis in the setting of rightsided IE. This case emphasizes the importance of recognizing the possibility of pulmonary septic emboli and other infectious foci in pulmonic valve endocarditis, especially in high-risk populations such as injection drug users.

\section{Data Availability}

Previously reported data were used to support this case report and are available within the manuscript. These prior studies are cited at relevant places within the text as references [1-15].

\section{Conflicts of Interest}

The authors declare that they have no conflicts of interest.

\section{References}

[1] L. Lopez, "The opioid crisis and the war on drugs," in The Color Atlas and Synopsis of Family Medicine, 3e, R. P. Usatine, M. A. Smith, E. J. Mayeaux, and H. S. Chumley, Eds., McGraw-Hill, New York, NY, USA, 2020, https://accessmedicine.mhmedical.com/content.aspx? bookid $=2547 \&$ sectionid $=206783206$.

[2] J. S. Alpert and S. A. Klotz, "Infective endocarditis," in Hurst's The Heart, 14e, V. Fuster, R. A. Harrington, J. Narula, and Z. J. Eapen, Eds., McGraw-Hill, New York, NY, USA, 2020.

[3] D. Swaminath, Y. Yaqub, R. Narayanan, R. F. Paone, K. Nugent, and A. Arvandi, "Isolated pulmonary valve endocarditis complicated with septic emboli to the lung causing pneumothorax, pneumonia, and sepsis in an intravenous drug abuser," Journal of Investigative Medicine High Impact Case, vol. 1, no. 4, 2013.

[4] S. B. Lieber, O. Tishler, K. Nasrullah, M. L. Fowler, R. H. Shmerling, and Z. Paz, "Clinical features of patients with septic arthritis and echocardiographic findings of infective endocarditis," Infection, vol. 47, no. 5, pp. 771-779, 2019.

[5] T. J. Cahill and B. D. Prendergast, "Infective endocarditis," Lancet, vol. 387, no. 10021, pp. 882-893, 2016.

[6] D. R. Murdoch, G. R. Corey, B. Hoen et al., "Clinical presentation, etiology, and outcome of infective endocarditis in the 21st century: the international collaboration on endocarditis-prospective cohort study," Archives of Internal Medicine, vol. 169, no. 5, pp. 463-473, 2009.

[7] S. Pant, N. J. Patel, A. Deshmukh et al., "Trends in infective endocarditis incidence, microbiology, and valve replacement in the United States from 2000 to 2011," Journal of the American College of Cardiology, vol. 65, no. 19, pp. 2070-2076, 2015.

[8] M. H. Crawford and S. Doernberg, "Infective endocarditis," in Current Diagnosis \& Treatment: Cardiology, 5e, M. H. Crawford, Ed., McGraw-Hill, New York, NY, USA, 2012, https:// accessmedicine-mhmedical-com.proxy.mul.missouri.edu/content. aspx?bookid=2040\&sectionid=152995671.

[9] S. M. Seraj, E. Gill, and S. Sekhon, "Isolated pulmonary valve endocarditis: truth or myth?" Journal of Community Hospital Internal Medicine Perspectives, vol. 7, no. 5, pp. 329-331, 2017.

[10] M. P. Ranjith, K. F. Rajesh, G. Rajesh et al., "Isolated pulmonary valve endocarditis: a case report and review of literature," Journal of Cardiology Cases, vol. 8, no. 5, pp. 161-163, 2013.

[11] A. Gomes, A. W. J. M. Glaudemans, D. J. Touw et al., "Diagnostic value of imaging in infective endocarditis: a systematic review," The Lancet Infectious Diseases, vol. 17, no. 1, pp. e1-e14, 2017.

[12] M. Saleem, F. Ahmed, K. Patel et al., "Isolated pulmonic valve endocarditis: case report and review of existing literature on diagnosis and therapy," CASE (Phila), vol. 3, no. 5, pp. 227-230, 2019. 
[13] A. Khosravi, Z. Rostami, M. Javanbakht et al., "Pulmonary endarteritis and endocarditis complicated with septic embolism: a case report and review of the literature," $B M C$ Infectious Diseases, vol. 20, no. 1, p. 212, 2020.

[14] K. Y. Kebed, K. Bishu, R. I. Al Adham et al., "Pregnancy and postpartum infective endocarditis: a systematic review," Mayo Clinic Proceedings, vol. 89, no. 8, pp. 1143-1152, 2014.

[15] W. D. Bolton, E. R. Fox, J. G. Winscott, and G. M. Aru, "Methicillin-resistant Staphylococcus aureus pulmonary valve endocarditis," The American Journal of Surgery, vol. 75, no. 12, pp. 1265-1266, 2009. 